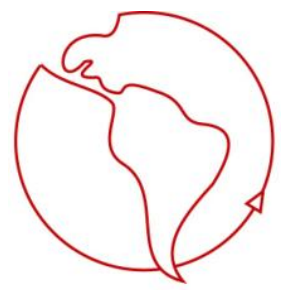

\title{
La Individuación del Alumno
}

Maximiliano Soto Sepúlveda

Magíster en Antropología y Desarrollo. Universidad de Chile

maxiclio@ hotmail.com

\section{Introducción}

Al problematizar la situación de la educación, en general, y de la relación profesor - alumno, en particular, dentro de sociedades de capitalismo periférico, como es el caso de Chile; nos planteamos la necesidad de entender las múltiples reacciones que se generan dentro y fuera de la sala de clases, una serie de variables que sólo se pueden develar a través de las conversaciones entre profesor y alumno, entre docente y discente. Es precisamente en esta situación que se construyen las identidades, en la medida que los alumnos se sienten participes de un sistema en particular.

Cuando la presente Reforma Educacional incluyó entre sus más repetitivos principios como el aprender a aprender, nos hizo sentir una gran preocupación conceptual. Nosotros concebimos la escuela como un espacio centrado en la comprensión de conceptos y realidades, en que nos distanciamos de la educación bancaria centrada en el almacenamiento de información, por lo tanto no puede hablarse de un aprender a aprender sino que de un aprender a comprender, a observar y a distinguir un mundo de otro.

Estas aclaraciones nos permiten dar curso a la estructura del presente trabajo, centrada en la condición de individuo (Alumno), en la escuela como un sistema de interacción, en el poder de las conversaciones, y en la construcción de identidades dentro de la sala de clases, con la preocupación de la cultura cívica actual. Este último planteamiento nos sustenta nuestra concepción de que ante la desaparición de la asignatura de Educación Cívica de los colegios (con la presente Reforma Educacional) sólo nos queda incluirla como un objetivo transversal y no con meros talleres o unidades de participación ciudadana dentro de la asignatura de Historia y Ciencias Sociales; creemos que la inclusión de esta temática es la que nos permitiría conocer y por tanto acceder a la comprensión del capital cultural de cada alumno, de las causas de identificación con tal o cual interés, de la causa de tal o cual decisión.

Además consideramos que el papel del profesor nos es solo de comunicador (como el comunicador radial) sino que de actor principal en el proceso de educación, entendida como una actividad humana de transformación y de transmisión cultural, pero de transformación sólo a través de la interpretación, de la comprensión que se inicia con la observación dentro del aula. El profesor hoy en día frente a los necesarios cambios debe abordar la inclusión en este sistema, en que la labor de investigador es crucial; por lo tanto no se deberían aceptar pedagogos investigadores fuera del sistema educacional, sino que dentro de él.

Los conceptos claves para entender y abordar la construcción de identidades dentro del sistema educacional se relacionan con un contexto económico, político y social, en que las sociedades periféricas que están excluidas de los grandes beneficios del primer mundo, deben sufrir adecuaciones forzadas frente a la globalización, y ciertos niveles de participación a modo de inclusión forzada; nos referimos al concepto de individuación como constructo de identidad social. Pero la pregunta central del análisis debe ser ¿Cómo el yo (profesor) acepta al otro (alumno)?, ¿Cómo lo comprende desde su yo?, ¿Cómo lo hace participe de su clase?, ¿Cómo se lo incluye desde su Otro?; son estas interrogantes las que trataremos de contestar.

\section{Conceptos Detrás del Individuo}


Entendido el alumno como un individuo dentro de un sistema particular como el educacional esta sometido a diversas situaciones de interacción y de comunicación con sus otros. Al intentar contestar la pregunta de ¿Cómo el alumno construye su identidad? o ¿Cómo se identifica desde su sí mismo con temas de interés personal?, hay que precisar conceptos como el de la identidad: “...identidad es siempre sistema. Su existencia no descansa en un núcleo inmutable del ser, al que tendría que encontrar el conocimiento, sino en la conservación de su función de orden para un experimentador constante, socialmente orientado" (Luhmann 1996:16) ${ }^{1}$.

En este sentido el alumno individuo sería parte de una estructura en particular denominada sistema, con una condición de ordenador a través de su identidad, desde donde se generarían redes comunicacionales que permitirían la interacción de unos con otros. Y en las relaciones de dicho individuo para con sus otros se genera un cuadro de múltiples interacciones, en donde el otro se proyectaría como parte del yo: "La existencia del otro sólo podrá ser afirmada más allá de la probabilidad, como total seguridad, si partimos de una experiencia inmediata e indudable, como la experiencia del cogito me asegura de mi propia existencia [...] Esta experiencia existe: es la vergüenza que me produce la mirada. La vergüenza como vivencia que es, $y$, por lo mismo, modo de conciencia, por ser de conciencia es intencional, referida a un objeto que no puede ser otro sino yo mismo, pero yo mismo, en cuanto aparezco ante otro, en cuanto soy visto por otro. La vergüenza me afirma al otro con total certeza. La mirada me reduce a objeto. Vivencio ser - mirado - objeto. Y esto es la vergüenza. No puedo ser objeto sino a otras consciencias. El otro es sujeto presente - sujeto en mi vivencia. En este sentido soy - para - otro" (Moreno 1995:271) $)^{2}$. Por lo tanto no se puede hablar de "uno" sin hacer referencia al "otro", ya que uno es uno mismo sólo en la medida de que hay uno otro, esa; y en ese otro hay una proyección del uno. En este sentido la construcción de la identidad sería tal sólo en la medida de que hay un otro; y es en el sistema educacional en donde se puede dejar claro la mirada de los observadores que identificaría dichas identidades.

Luego de dejar señalado, nuestros paradigmas conceptuales de la identidad y del uno frente a un otro, cabe señalar el marco de la construcción de identidades dentro de la situación particular de nuestra sociedad frente a ciertos contextos de carácter periférico. Cuando hablamos de individuación nos referimos a la construcción del individuo en un contexto de capitalismo periférico: “...la dinámica de las sociedades periféricas si bien generan relaciones de interdependencia y asimetría con el resto de las naciones desarrolladas en el mundo contemporáneo y globalizado, significan además relocalización y deslocalización. Deslocalización en la medida en que la globalización afecta singularmente desde afuera a los países de la periferia, y relocalización porque para que la globalización funcione efectivamente, debe convertir lo que proviene desde afuera, en un componente de la cultura local : por lo tanto, el capitalismo periférico no es la imagen del espejo retrovisor del capitalismo desarrollado con estado de bienestar, sino que es lago particular, que se mueve sincrónicamente a aquél, pero no detrás de él tratando de alcanzarlo" (Robles 1999:294) ${ }^{3}$.

Es frente a esta situación que la construcción de identidades dentro de estas sociedades no es sólo un constructo social con un claro matiz de atraso económico, sino que es un mundo cultural totalmente nuevo y distinto, en donde las identidades que se van forjando no son de carácter contestatario y reaccionario, sino que dan forma a individuos únicos a través de todo un proceso de individualización en lo que Niklas Luhmann llama Las Sociedades de Riesgo, lo que implica una doble entrada en el sistema de sociedad . Es desde este punto de vista que podemos entender, en el caso del sistema educacional, la falta de compromiso con temas políticos o socioeconómicos, la falta de asistencia laboral, la falta de aceptación al otro como otro, la falta de espacialidad de los discursos. La individuación como proceso de

\footnotetext{
${ }^{1}$ Luhmann, Niklas. Teoría de la sociedad y pedagogía Ed. Paidos , Barcelona, 1996, c 1992, pág.16

${ }^{2}$ Moreno, Alejandro. El aro y la trama. Episteme, modernidad y pueblo, CIP, Caracas, 1995, pág.271

${ }^{3}$ Robles, Fernando. Los sujetos y la cotidianeidad. Elementos para una microsociología de lo contemporáneo. Ed Sociedad Hoy, Talcahuano, 1999, pág.294.
} 
individualización genera los siguientes impactos: “...la autoconfrontación consigo mismo en medio de unidades específicas de socialización, en el caso de la individualización es un proceso de autoconfrontación asistido, mientras que en el capitalismo periférico se trata de una autoconfrontación desregulada, y significa por lo tanto un aumento significativo de las inseguridades ontológicas condicionadas por el empleo precario, la subcontratación, las antinomias de las relaciones de trabajo, las diferencias de género, etc." (Robles 1999:300) ${ }^{4}$. En esta afirmación se plantea que la gran diferencia conceptual entre la individulalización y la individuación radica entre: un "Haz de tu vida lo que quieras" y un "Arréglatelas como puedas", en relación a una clara diferenciación entre inclusión y exclusión.

En este planteamiento hay una íntima relación entre el yo (uno) y el mi (otro), ya que cuando se refiere a la "autoconfrontación desregulada", se refiere a una confrontación del sujeto para consigo mismo, del individuo frente a su entorno, entendido como su otro; por lo tanto necesariamente tiene que referirse a su otro, tiene que buscarlo en un contexto de ruptura de reciprocidades. " ... la identidad de una construcción tal, deja de ser, pues, una connotación referida a estados interiores del sujeto para adquirir validez en la medida en que se exterioriza, porque pasa progresivamente en el contexto de la evolución constitutiva de la dialéctica del Yo y el Mi, a ser dependiente del reconocimiento de los destinatarios [...] El paso del Yo al Mi sólo es posible mediante la interacción :precisamente es allí donde la individualización y la individuación se bifurcan" (Robles 1999:301) ${ }^{5}$. En este sentido el sujeto en las sociedades del capitalismo central busca al otro por un querer, desear y escoger, a modo de opción; mientras que en las sociedades del capitalismo periférico, el sujeto esta obligado a buscar al otro, esto marca la diferencia entre una autoconfrontación asistida y una autoconfrontación desregulada. "La gran diferencia entre la individualización y la individuación reside allí donde las inseguridades manufacturadas alas que están expuestos los que viven en la periferia globalizada son doblemente más determinantes para la composición de la biografía individual y para la construcción de las estructuras cognitivas: la autoconfrontación del sujeto consigo mismo es desregulada y no asistida (por nadie), pero al mismo tiempo la búsqueda del otro es obligada y no escogida. Todo esto hace que el Mí conservador pueda ser obligado a desplazar al Yo en el rol revolucionario"6.

En relación a este cuadro de interacciones es que consideramos la posición dentro del sistema educacional de dos actores claves, profesor - alumno, docente - discente, como una sustentación de la obligación de buscar al otro, en un sentido bilateral, y que la apatía y desinterés frente a temas contingentes radica en una identificación fragmentada y desregulada. Creemos que la única forma de interacción asistida en sociedades del capitalismo periférico radica en una comprensión interaccional entre profesor - alumno, en la descripción e interpretación de los varios capitales culturales al interior del aula de clases.

\section{Educación y Comprensión}

Para comenzar a enfatizar esta necesaria y apremiante interacción es de primera importancia tener claramente señalado nuestro concepto de educación, y criticar esa continua y desgastante situación del aprender a aprender centrado en la información. “...proceso de inducción al conocimiento relacionado con las cosas importantes para la vida, sus bienes intrínsicos pueden clasificarse de acuerdo con las tres dimensiones interrelacionadas del desarrollo personal: intelectual, moral y espiritual. Los bienes intelectuales consisten en el desarrollo de las potencias de la comprensión humana en relación con los problemas vitales. Los bienes espirituales consisten en el desarrollo de la sabiduría a través del ejercicio de estas potencias intelectuales, y los bienes morales consisten en las virtudes o actitudes que son

\footnotetext{
${ }^{4}$ Ibidem,pág.300

${ }^{5}$ Ibidem, pág.301

${ }^{6}$ Ibidem, pág.307
} 
condiciones necesarias para el desarrollo de las potencias de comprensión y del descubrimiento de soluciones a los problemas fundamentales de la vida" (Eliott 1990:300) ${ }^{7}$.

En este concepto la hermenéutica constituye el marco de acción a seguir, ya que a través de las interpretaciones de carácter inductivo se puede llegar a develar el capital cultural de los individuos, sus múltiples mundos, su biografía personal participes de este proceso, y de estas interpretaciones se puede llegar a la transformación de la sociedad, no sólo sustentada en una teoría de la acción (Max Weber), sin que el elemento del lenguaje y de las comunicaciones debe ser incluido en este proceso (Rafael Echeverría), en donde se aplique un entendimiento global y a la vez particular de este sistema de sociedad.

Es desde esta perspectiva que criticamos la simple entrega de información que vela por un saber deductivo y no inductivo en este proceso de transformación. "Desde este punto de vista no basta con transmitir a los estudiantes informaciones inertes sobre ideas, conceptos y teorías en forma de definiciones. En el proceso de inducción, el profesor los representa como normas críticas que traslada el pensamiento de los estudiantes sobre los problemas de la materia. Están implícitos en las cuestiones que plantea, en la evidencia sobre la que llama la atención y en las pruebas a las que impide que los estudiantes sometan su pensamiento. Es más, el profesor debe inducir a los estudiantes de forma que les proporcione el acceso a la naturaleza problemática y discutible de las estructuras de conocimiento. Un proceso aceptable, desde el punto de vista educativo, supone una actitud reflexiva tanto de parte del profesor como de los estudiantes. El diálogo entre el profesor y los alumnos y entre los propios estudiantes constituye principios de procedimiento que rigen cualquier inducción educativa al conocimiento" (Eliott 1990: 292$)^{8}$.

Es en torno a esta actitud reflexiva que se va gestando una interacción entre los participes del proceso educativo; desde donde se inician las transformaciones a nivel de la comprensión. En lo cual el diálogo es crucial para dar inicio a esta inducción del pensamiento, no sólo a nivel de profesor - alumno, sino que también entre alumno - alumno; es a este nivel en donde el individuo “... tiene la posibilidad de intensificar, en ciertos casos, sus relaciones personales, de comunicar a otros algo de lo que le es íntimamente propio, buscando así su confirmación" (Luhmann 1985: 13) ${ }^{9}$. Es a través del diálogo en donde el uno se proyecta en el otro, es a través del uso de la palabra que se genera la proyección y descubrimiento de lo íntimo. "En las relaciones sociales el impulso personal no puede extenderse, sino que intensificarse [...] ese impulso hace posible unas relaciones sociales personales en las que destacan ciertas cualidades individuales $\mathrm{o}$, preferentemente, todas las cualidades de una persona individualizada" (Luhmann 1985:15) ${ }^{10}$.

Pero ¿Cómo se genera la comprensión dentro del sistema educativo?, ¿Cómo se producen los distintos intercambios vivenciales en el marco interaccional profesor - alumno? Para contestar estas interrogantes es necesario remitirse al texto de Niklas Luhmann, Teoría de sociedad y pedagogía, y de esta manera entender como opera la comprensión sustentada en la observación dentro del sistema educacional, y dentro del aula de clases: “...la comprensión sólo es posible cuando el que en cada momento es el otro se mantiene a la vista [...] que el sistema comprensivo pueda experimentarse a sí mismo como un momento en el entorno del sistema comprendido. El sistema comprensivo se identifica entonces por así decirlo en una doble reducción: como uno entre muchos otros en el entorno de un sistema entre muchos otros en su entorno" (Luhmann 1996: 101) ${ }^{11}$. Distingue entre lo sistemas comprendidos y los sistemas comprensivos, y dentro de esta distinción el sistema educacional, al igual que el sistema psíquico, tiene la capacidad de observar a otros sistemas, ahí radica la distinción del epistema. Por lo tanto el sistema educacional sería un sistema societal que tendría la capacidad de autoobservarse y por tanto autocomprenderse, y a la vez observar su entorno sistémico.

\footnotetext{
${ }^{7}$ Eliott, John. La investigación - acción en educación. Ed Morata, Madrid, 1990, pág.300

${ }^{8}$ Ibidem, pág. 292

${ }^{9}$ Luhmann, Niklas. El amor como pasión. Ed Península, Barcelona, 1985 c 1982, pág.13.

${ }^{10}$ Ibidem pág 15

${ }^{11}$ Op.Cit.Luhmann. Teoría de la sociedad y pedagogía, pág.101.
} 
Desde la praxis aula de clases los alumnos tendrían la capacidad de, frente a una situación en particular como de desorden, observar la actitud del profesor, la de sus compañeros, y la de la situación y desentenderse o identificarse con tal. En este sentido no podemos seguir afirmando una comprensión unilateral, dese el profesor a los alumnos, sin entender los códigos que se vierten en las múltiples interacciones comunicacionales que se generan entre los distintos participes de la clase, los alumnos. Pero estas relaciones son difíciles de considerar dentro de una educación que prima por la información o por la compresión unilateral de los contenidos, de los textos. “...el problema de la comprensión se podía reducir a la tarea de entender si el alumno había comprendido el conocimiento a adquirir. Se trataba pues de una relación de textos y cabezas. El apropiarse de los textos estaba pensado como confirmación de la autorreferencia [...]. Por consiguiente, la educación estaba considerada para aprender a comprender el mundo por cuenta del alumno. Pero nadie dudaba seriamente de que el profesor pudiera controlar y en su caso corregir la comprensión del texto por el alumno [...] La abrupta reducción del problema de la comprensión a un problema cabeza - texto ha de ser en todo caso abandonada, ha de traslucirse como mínimo en una mentira piadosa profesional" (Luhmann 1996:94) ${ }^{12}$.

En este planteamiento se hace una dura crítica a la pedagogía neo humanista que puso en práctica la educación diferenciada; poniendo en duda la incorporación, en la comprensión de los textos, de la biografía personal de los alumnos, de sus capitales culturales, en contra de una unidireccionalidad en la comprensión del mundo y de los problemas societales, que no considera la comprensión, interpretación y observación de los alumnos en su condición de observadores autorreferenciales. "El sistema social clase observa y comprende a los alumnos desde el punto de vista de la diferencia entre comprender y no comprender [...] Pero la clase sólo entiende la comprensión o no comprensión cuando puede observar el nivel de información y las comunicaciones en marcha con la autorrefencia del alumno" (Luhmann 1996:135 $)^{13}$.

\section{Profesor y Alumno}

La interacción entre los profesores y los alumnos se sustenta en la observación que deriva en la comprensión dentro del sistema educacional. Por lo tanto en el sistema educativo: “...el profesor tiene que observar paso a paso lo que los alumnos observan, y esto en una situación en la que los alumnos están más o menos obligados a observar al profesor. De este modo, el profesor se ve forzado por los alumnos a la autoobservación, y por eso a la pedagogía parece bastarle al principio con ocuparse del profesor y de sus métodos" (Luhmann 1996:45-46) ${ }^{14}$.

Si bien se genera un cuadro de múltiples observaciones entre el profesor y los alumnos, y entre estos mismos, el problema de la comprensión de la educación radica en una falta de inclusión de los alumnos como individuos autorreferenciales dentro del sistema educacional, lo que se evidencia en una comprensión unidireccional de los problemas. “...dado que la clase sólo se puede poner en marcha como sistema de interacción de profesores y alumnos, los problemas iniciales de los niños se convierte en problemas del profesor, y por tanto en problemas sistemáticos del sistema educativo..." (Luhmann 1996:44) $)^{15}$. Y el gran problema que se genera dentro de la sala de clases es que no se puede aplicar una educación homogeneizada para todos igual, ya que hay diferencias de mundos, de biografías personales, de experiencias de vida diferentes, en donde el profesor que lleva bastantes años haciendo clase simplemente aplica su rutina pedagógica aceptando y tratándose comprender los problemas individuales, y para él los alumnos son todos iguales, ya que todos tienen problemas; pero los alumnos están en un mundo totalmente nuevo cuando al profesor es una persona que nunca han visto. "Para el alumno, el comienzo de

\footnotetext{
12 Ibidem, pág.94

${ }^{13}$ Ibidem pág. 135

${ }^{14}$ Ibidem pág.45-46

15 Ibidem pág.44
} 
la escuela trae consigo una ruptura en su ritmo de vida, para el maestro en todo caso una nueva generación de alumnos con características ligeramente distintas. El alumno tiene que superar el problema sin las experiencias previas pertinentes, y al mismo tempo mucho de su futuro depende de cómo resuelva el problema. El maestro sabe ya lo que hay que hacer, y en todo caso aprende mínimamente de ello. No es pues para el alumno, sino para el maestro, para quien se trata de un "comienzo homogeneizado" y una repetida aplicación de reglas. El alumno tiene que superar una discontinuidad individual en su forma de vida" (Luhmann 1996:58) ${ }^{16}$.

Es en este sentido que los alumnos concebidos como individuos dentro de un sistema, intentan dar solución a sus problemas al interior de dicho sistema educacional en una situación de individuación, de una autoconfrontación desregulada en la que el alumno tiene la obligación de dirigirse hacia su otro, profesor, sin un planteamiento de un querer, o desear, en relación a lo que podría ser una autoconfrontación asistida. Y es frente a esta situación que se generan apatías, suertes de identidades fragmentadas, una rigurosa vinculación de los textos a la cabeza, que no dejan espacios para varias interpretaciones y comprensiones, en donde hay una clara ausencia de la incorporación de las observaciones de los alumnos dentro del sistema educacional, como podría ser a manera de generar estrategias de aprendizaje desde su observación, como ente sistémico, dando espacios a los intercambio ilocucionarios sustentados en el diálogo, para que permiten una transformación a través de las interpretaciones comunicacionales.

\section{Escuela y Familia}

En relación a la interacción que se genera entre profesor y alumno hay que señalar la clara diferencia que éste último experimenta con respecto a su familia, y su capacidad de observación como medio de distinción y de comprensión: “...la educación en el sistema de interacción enseñanza se diferencia esencialmente de la educación en las familias; y ello porque la observación y comprensión se dirige de otro modo. En las familias, todas las diferencias son individuales [...]. La persona se individualiza como referencia respecto a los otros" (Luhmann 1996:117) ${ }^{17}$.

Desde esta posición los individuos dentro de la familia experimentan una identidad compactada y no fragmentada, en donde habría una autoconfrontación asistida, y que al momento de pasar el alumno al aula de la sala de clases se desrregulariza, entrando en una necesidad de encuentro obligado hacia el otro. En este sentido habría una mayor tolerancia de las diferencias al interior de las familias, y no así al interior del aula: “...debido a una relevancia personal hay una mayor tolerancia para las particularidades, defectos, autocontroles defectuosos de los padres educadores, que en el caso del maestro en la escuela. Consecuentemente, las peculiaridades de los maestros están expuestas al ridículo en mayor medida que las peculiaridades del padre o de la madre, hay en el colegio una muy característica problemática de la conducta evitadora del ridículo que falta por completo en la familia" (Luhmann 1996:118) ${ }^{18}$.

Dentro del sistema familia el individuo comprende desde su condición individual, es desde ahí desde donde establece distinciones, y que son aceptadas en cuanto tal ya que desde ellas construye su identidad en un circuito de pertenencia, lo que sería completamente diferente al sistema clase, en donde el individuo esta obligado a establecer distinciones de comprensión según el profesor. En la escuela: “...el aprender a comprender se basa pues en la comprensión, pero en u sentido muy especializado. Se trata de comprender la información mediante una comprensión del profesor. El alumno tiene que poder entender cómo entiende el profesor, y a menudo incluso cómo consigue comunicárselos ahora y en una determinada forma [...]. Sólo tiene que entender que el profesor emplea su persona para llevar a cabo su

\footnotetext{
${ }^{16}$ Ibidem, pág.58

${ }^{17}$ Ibidem pág. 117

${ }^{18}$ Ibidem pág. 118
} 
papel, que se ha preparado especialmente bien, que ama sus propios conocimientos, se entusiasma con ellos..." (Luhmann 1996:120) ${ }^{19}$.

Es precisamente en esta diferencia en donde se desarrolla la condición de individuación de las identidades fragmentadas propias de los individuos pertenecientes a las "sociedades de riesgo", en un contexto de capitalismo periférico. Ahí es donde los individuos entran en un contexto de identificación obligada hacia el otro, y ahí radican los múltiples conflictos que se generan entre los profesores y los alumnos. Lo que se visualiza en materias de profunda repercusión como la educación cívica, en cuanto a lo cual ellos generan una postura desinterezada, ya que no se identifican con tal o cual temas, en este cuadro se han involucrado obligadamente, es así como lo sientes. Es en un acercamiento al diálogo donde están las soluciones de interacción constante para generara una situación de amplias interpretaciones, en el sistema educacional se hace necesario una inclusión de las comprensiones de mundo, desde el individuo, y no comprensiones impuestas desde los otros, los profesores. Es sólo en una condición dialógica de vida en donde se hace posible un aprender a comprender, para luego transitar a un aprender a vivir.

Por lo tanto vemos una cierta condición de exclusión en las distinciones que hace el alumno dentro de la escuela y de la sala de clases, no se aceptarían sus formas de comprender la información desde su individualidad, y existiría una suerte de comprensión impuesta. "La escuela excluye como siempre, pero en lo sucesivo lo hace de manera continua, en todos los niveles del curso (entre las clases de transición y los liceos de enseñanza técnica tal vez no haya sino una diferencia de grado), y conserva en su seno a quienes excluye, contentándose con relegarlos a las ramas más o menos desregularizadas. De ello se deduce que esos excluídos del interior están condenados a moverse, sin duda en función de las fluctuaciones y oscilaciones de sus sanciones, entre la adhesión entusiasta a la ilusión que propone y la resignación a sus veredictos, entre el sometimiento ansioso y la rebelión impotente. No pueden dejar de descubrir, más o menos pronto, que la identidad de las palabras ocultas, la diversidad de las cosas: que el establecimiento en que los colocó la orientación escolar es un lugar de reagrupamiento de los más desprovistos..." (Bourdieu 1999:366) ${ }^{20}$. En este planteamiento que desarrolla el sociólogo Pierre Bordieu queda claramente señalado una suerte de desorientación por exclusión de las individualidades, de las biografías personales, lo que afectaría en gran medida el desarrollo de la educación superior, lo que pondría en tela de juicio el papel de los profesores en la orientación actual: “...la organización actual del sistema de enseñanza no permite que la docencia brinde a los alumnos una asistencia intensiva y diferenciada; [...] ésta se torna indispensable con el progresivo incremento de la proporción de estudiantes poco provistos de un capital cultural, los cuales - por esa razón - tienen más que aprender de la escuela" (Bourdieu 1999: 396) ${ }^{21}$.

Hay que insistir en lo apremiante del establecimiento de espacios, dentro del sistema educacional, que permitan el desarrollo de una nueva educación de carácter dialógica que posibilite el desarrollo de los alumnos, el destape y profundización de sus capitales culturales, una inclusión de sus observaciones, de sus distinciones, y en definitiva de sus maneras de comprender el mundo que les rodea. Es solo a través de un diálogo profundo y constante en la interacción entre profesor - alumno que se puede llegar a una educación que vele por el aprender a comprender y el aprender a vivir, desde donde se genere una verdadera orientación educacional, no sustentado en las comprensiones de mundo impuestas por el profesor.

Es necesario considerar que la educación como actividad humana es una transmisora de patrones culturales, de forma de comportamientos, de habitus de vida, de biografías personales. "La educación es un proceso mediante el cual la comunidad transmite su cultura y las personas se forman y desarrollan sus capacidades para participar plenamente de la vida en común. [...]. Sujeto de la educación es una persona, del todo particular, provista de derechos y deberes; un individuo de naturaleza genérica, que comparte con

\footnotetext{
${ }^{19}$ Ibidem pág. 120

${ }^{20}$ Bourdieu, Pierre. La miseria del mundo. Ed FCE, Buenos Aires, 1999, pág.366

${ }^{21}$ Ibidem, pág. 396
} 
sus semejantes y que es, sin embargo, de naturaleza completa, única e irrepetible, convocación y proyectos propios" (Comité asesor del diálogo sobre la modernización de la educación chilena, 1995:21) ) $^{22}$.

\section{El Poder de las Conversaciones}

En los puntos anteriores hemos planteado que los individuos construyen su identidad desde una situación contextual, y que ellos mediante observaciones van distinguiendo y comprendiendo su mundo. Ahora nos cabe señalar cuál es el papel y la importancia real del diálogo y de las conversaciones entre los actores principales dentro del sistema educacional, el profesor y el alumno.

Es precisamente a través de estas instancias de comunicación que se generan las interpretaciones acerca del mundo que nos rodea, desde donde aceptamos y comprendemos nuestro mundo como una realidad existente. Por lo tanto las cosas existirían, como tales, sólo en nuestras interpretaciones de ellas: "Pensemos que nunca podemos decir cómo las cosas realmente son: sólo podemos decir cómo nosotros las interpretamos o consideramos" (Echeverría 1997:40) ${ }^{23}$. El filósofo chileno Rafael Echeverría plantea que la existencia del ser de las cosas radica en el lenguaje, ya que desde ahí le damos su existencia, con lo que las denominamos, las describimos, y las presenciamos.

Desde esta perspectiva, la interacción que se genera entre el profesor y el alumno es crucial para dar existencia a la individualidad y a las biografías personales, a las experiencias de vida, a los capitales culturales, ya que sino se internalizan a través del lenguaje dichos mundos, y dichas formas de comprensión no existen. Es sólo desde este entendimiento conceptual que las comprensiones de mundo desde la individuación del alumno pueden ser incluidas en el sistema educacional; ahí radica la importancia del diálogo y de que se generen espacios conversacionales.

En esta interacción es primordial el acto de hablar, pero también el de escuchar, ya que es ahí donde se diferencia el diálogo del discurso, es en el escuchar que el otro se vincula con el uno, el Yo con el MI. "Si examinamos detenidamente la comunicación, nos daremos cuenta, de que ella descansa, principalmente, no en el hablar sino en el escuchar. El escuchar es el factor fundamental del lenguaje. Hablamos para ser escuchados. El hablar efectivo sólo se logra cuando es seguido de un escuchar efectivo. El escuchar valida el hablar. Es el escuchar, no el hablar, lo que confiere sentido a lo que decimos. Por lo tanto, el escuchar es lo que se dirige. Por lo tanto, el escuchar es lo que dirige todo el proceso de la comunicación" (Echeverría 1997:142) ${ }^{24}$.

Hay que hacer ciertas diferencias en relación a la precisión conceptual, ya que en la praxis educacional el escuchar a los alumnos se diferencia notablemente del sólo oírlos. "Lo que diferencia el escuchar del oír es el hecho de que cuando escuchamos, generamos un mundo interpretativo. El acto de escuchar siempre implica comprensión y, por lo tanto, interpretación. Cuando atribuimos una interpretación a un sonido pasamos del fenómeno del oír al fenómeno del escuchar. Escuchar es oír más interpretar. No hay escuchar sino hay involucrada una actividad interpretativa. Aquí reside el aspecto activo del escuchar..." (Echeverría 1997:146) ${ }^{25}$.

Es a través de estas instancias de comunicación que se generan una serie de intercambio lingüísticos que permiten la interacción entre el profesor y los alumnos, desde donde se construye una relación no impuesta, dando espacio a una autoconfontación asistida. "... nuestras conversaciones generan el tejido en el que nuestras relaciones viven. Las conversaciones y las relaciones son una misma cosa. Mantendremos una relación con alguien mientras estemos en una conversación abierta y continua con esa

\footnotetext{
${ }^{22}$ Comité técnico asesor del diálogo nacional sobre la modernización de la educación chilena. Los desafíos de la educación chilena frente al siglo XIX. Ed Universitaria, Santiago, 1995, pág.21

${ }^{23}$ Echeverría, Rafael. La ontología del lenguaje, Ed Dolmen, Santiago, 1997 c 1994,pág.40

${ }^{24}$ Ibidem pág. 142.

${ }^{25}$ Ibidem pág. 146
} 
persona. Es eso lo que define una relación. Sí, por cualquier razón, la conversación se interrumpe o termina, la relación también se interrumpe o termina" (Echeverría 1997:244) ${ }^{26}$

Desde esta posición comprendemos las conversaciones como relaciones comunicacionales que se generan entre las personas, y que en la existencia de estas situaciones recaen las interacciones permanentes entre el profesor y el alumno. Por lo tanto una individualidad deseada y asistida, depende de la existencia de estas instancias al interior del aula de clases; sólo de esta manera se podrá implementar una educación que acepte y optimice las diferentes formas de comprensión e interpretación del mundo.

\section{Cultura Cívica}

Nuestra preocupación central recae (como ya lo hemos señalado) en la falta de interés de los alumnos de enseñanza media por aspectos del orden cívico, por temas de la preocupación ciudadana, por un falta de identificación con el espacio público. Situación que se suma a la apatía del alumno por conversar con el profesor, o por participar en clases; lo que consideramos que se debe a una situación de la individuación del alumno en la construcción de su identidad, caracterizada por una autoconfrontación desregulada del "uno" en su relación con los "otros" (Robles 1999).

Creemos que la situación de apatía y desinterés del alumno por una participación y preocupación cívica se debe tanto a un contexto global (Sociedades del capitalismo periférico), como a un contexto particular (en cuanto a la situación por la que esta pasando la política chilena): "La falta de crítica al modelo ha sido útil para crear consensos entre gobierno y oposición, ha minimizado los conflictos entre los partidos, pero también ha hecho aparecer a la política como una actividad sin mayores sorpresas y, por lo tanto, menos interesante para la masa ciudadana que no participa activamente en ella. Por su parte, los marginados de los beneficios del modelo, y los que lo rechazan por razones ideológicas o morales, se marginan de toda actividad política o expresan su rechazo de forma atomizada, inorgánica y sin proponer alternativas que puedan ser acogidas por agrupaciones más fuertes" (Urzúa 1995:166) ${ }^{27}$. Desde esta postura se podría reflejar una instancia de exclusión controlada en cuanto a la participación cívica de la ciudadanía, por una proyección de no causar sorpresa y fomentar un seudoconsenso.

Se sabe que es muy difícil fomentar una discusión entre todas las partes o componentes de una sociedad, debido a un sentido de temor a las críticas desde ésta hacia las políticas de gobierno. Pero para poder asistir a ese sentimiento de proyección obligada del uno hacia los otros, de autoconfrontación desregulada, de la construcción de identidad propia del individuo, se hace necesario profundizar los diálogos y las instancias conversacionales, una actitud que a nuestro entender debe iniciarse en la educación, como instancia básica de transmisión y transformación cultural

Hay que entender que en todas las sociedades existen desigualdades, y que en relación a éstas se construyen nuevos mundos e imaginarios culturales distintos, comprensiones e interpretaciones de la realidad, y distintas formas de distinciones y percepciones "Así como no hay sociedades totalmente igualitarias, no hay tampoco sociedades en las cuales no existan normas que permitan distinguir entre "nosotros" y "los otros". Son esas normas las que transforman diferencias externas en criterios para establecer desigualdades acumulativas y de carácter más general. Es también la tendencia a la exclusión de los otros, los diferentes, la que lleva a que grupos sociales desigualmente colocados en la estructura de poder, en la división del trabajo y en el acceso a bienes y servicios, desarrollen subculturas propias, formas de distinción y al mismo tiempo de integración" (Urzúa 1995:157) ${ }^{28}$.

Es por lo tanto en la identificación de las diferencias donde se generan espacios de inclusión de los unos con respecto a los otros, lo que se profundiza a nuestro entender en el quehacer de las

\footnotetext{
${ }^{26}$ Ibidem, pág. 244

${ }^{27}$ Urzúa Raúl. Cultura cívica, desigualdad y equidad en Chile. Revista de Sociología. Departamento de Sociología, Universidad de Chile, Santiago, 1995.

${ }^{28}$ Ibidem, pág. 157
} 
conversaciones, de los diálogos, a través de los cuales de develan las distintas comprensiones e interpretaciones de mundo.

\section{Conclusiones}

1) La identidad del alumno se construye desde su situación de individuación, propias de las sociedades del capitalismo periférico.

2) Hay dos contextos a tener en cuenta en el análisis de la situación señalada en el punto anterior, a modo de efectos de la globalización, por un lado, la deslocalización y por otro, la relocalización, en cuanto a los efectos externos, como a las adecuaciones internas.

3) Las sociedades de riesgo constituyen constructos culturales y societales totalmente propios, no son formas culturales rezagadas del capitalismo central, de las sociedades industrializadas.

4) El proceso de Reforma Educacional propaga el aprender a aprender, lo que fomenta la exclusión dentro del aula de clases. Planteando un aprender a comprender se superaría la exclusión a favor de una inclusión de las distintas comprensiones de mundo.

5) Hay una clara diferencia entre la comprensión que se genera al interior de la sala de clases y la que se genera al interior de la familia. En la familia se generaría un autoconfrontación asistida, y en la escuela un autoconfrontación desregulada, que no aúna por la diversidad de criterios en la comprensión e interpretación de la realidad.

6) La escuela no fomenta el espacio de las conversaciones entre los participantes del proceso educativo, profesores y alumnos. Es en las conversaciones y en las instancias de diálogo donde se puede fomentar e implementar una autoconfrontación asistida que devele los capitales culturales a favor de un "Haz lo que quieras", y no un "Arréglatelas como puedas".

7) Hay un claro desinterés cívico en los estudiantes secundarios debido a una falta de identificación, a una exclusión de comprensiones de mundo, a la instauración de una política sin sorpresa, y a la presencia de un pseudoconsenso entre las partes opositoras.

8) Es en la distinción del otro, como otro en donde el Yo devela su individualidad, su capital cultural, su pertenencia e inclusión, desde donde se generan las identificaciones, comprensiones e interpretaciones de mundo.

\section{Bibliografía}

Bourdieu, Pierre. La Miseria del Mundo. Ed. Fondo de Cultura Económica, Buenos Aires, 1999.

Comité Técnico asesor del diálogo nacional sobre la modernización de la Educación chilena. Los Desafíos de la Educación chilena frente al siglo XXI. Ed. Universitaria, Santiago, 1995.

Echeverría, Rafael. Ontología del Lenguaje. Ed. Dolmen, Santiago, 1997 c 1994.

Eliott, John. La Investigación - Acción en la Educación. Ed. Morata, Madrid, 1990.

Luhmann, Niklas. El Amor como Pasión. Ed. Península, Barcelona, 1985 c 1982.

Luhmann, Niklas. Teoría de la Sociedad y Pedagogía. Ed. Paidós, Barcelona 1996 c 1992.

Moreno, Alejandro. El Aro y la Trama. Episteme, Modernidad y pueblos, CIP, Caracas, 1995.

Robles, Fernando. Los Sujetos y la Cotidaneidad. Elementos para una microsociología de lo Contemporáneo. Ed. Sociedad Hoy, Talcahuano, 1999. 
Urzúa, Raúl. Cultura Cívica, Desigualdad y Equidad en Chile. Revista de Sociología. Deptp. de Sociología, Universidad de Chile, Santiago, 1995. 